\title{
Dosagem da heparina em cirurgia cardíaca com circulação extracorpórea
}

\author{
Fernando MORAES ${ }^{*}$, Helena NADER ${ }^{*}$, Carlos P. DIETRICH ${ }^{*}$, Énio BUFFOLO* \\ RBCCV $44205-310$
}

Moraes F, Nader H, Dietrich C P, Buffolo E - Dosagem da heparina em cirurgia cardiaca com circulaçāo extracorpórea. Rev Bras Cir Cardiovasc 1996; 11 (3): 201-7.

RESUMO: Com o objetivo de quantificar o nivel sérico de heparina, sua atenuaçăo em funçăo do tempo - o valor residual após a neutralização com sulfato de protamina, foram coletadas amostras de sangue em tempos pré-estabelecidos em 27 pacientes submetidos a revascularizaçāo do miocárdio com circulaçāo extracorpórea. Após a heparinizaçăo (400 Ul/kg) as amostras de sangue foram coletadas nos tempos de 5 , 10,30 e 60 minutos e subseqũentemente a cada 30 minutos, dependendo do tempo da circulação extracorpórea. A cada hora, após a heparinização, administrava-se nova dose de heparina (200 Ul/kg). As amostras eram armazenadas à temperatura de $4^{\circ} \mathrm{C}$ antes do processo de extraçăo da heparina que foi realizado por métodos físico-químicos. As dosagens mostraram que 5 minutos após a heparinizaçāo os pacientes apresentaram concentração sangûinea máxima de heparina e, ao tempo de 60 minutos, a sua concentraçăo é de aproximadamente $68 \%$ da encontrada aos 5 minutos. Ao tempo de 90 minutos, ou seja, após a reheparinizaçāo a concentraçāo de heparina é $96 \%$ da evidenciada aos cinco minutos e, após a neutralização com sulfato de protamina $(1,5: 1)$, ainda se encontra um valor residual de heparina que corresponde a $4 \%$ do observado inicialmente. Observou-se que os pacientes mais idosos te̊m uma tendência a manter um nível sérico mais prolongado e através da equação (Conc. de heparina $=104,7+(-12,85 \times$ minutos (In)) $+0,25 \times$ idade) podemos estimar a concentraçăo de heparina em determinado tempo.

DESCRITORES: Heparina, sangue. Circulação extracorpórea. Revascularização do miocárdio. Heparina, cirurgia cardiaca.

\section{INTRODUÇĀO}

A heparina foi descorberta em 1916 por McLean, nos Laboratórios de Fisiologia da Universidade John Hopkins (1) e passou a ser utilizada em larga escala com o advento da circulação extracorpórea (CEC) no inicio dos anos 50 . Apesar de ser usada rotineiramente, uma série de dúvidas ainda persiste com relaçāo a dose adequada, a sua quantificaçāo sérica bem como a sua neutralizaçăo.

Para promover sua açăo anticoagulante a heparina, quando em quantidade suficiente, liga-se com grande afinidade à antitrombina inibindo, assim, uma série de serino-proteases envolvidas na cascata da coagulação (Fator Xlla, Xla, IXa, Xa e trombina) (2).

Em boa parte dos centros de cirurgia cardiaca - efeito anticoagulante da heparina é monitorizado através do Tempo de Coagulação Ativado (TCA), método descrito por HATTERSLEY, em 1966 (3).

BULL et al. (1) coletaram mais de 30 protocolos diferentes de equipes que realizam cirurgia cardíaca nos Estados Unidos. Selecionaram 5 protocolos, dos 30 analisados, para avaliar como se comporta-

Trabalho realizado na Disciplina de Cirurgia Cardiovascular do Departamento de Cirurgia e Departamento de Bioquimica da Escola Paulista de Medicina da Universidade Federal de Săo Paulo. Såo Paulo, SP, Brasil.

Apresentado ao $23^{2}$ Congresso Nacional de Cirurgia Cardiaca. Recite, PE, 20 a 23 de março, 1996.

- Da Escola Paulista de Medicina.

Endereço para correspondẻncia: Fernando Moraes. Av. Bernardo Vieira de Melo, 4405/401. Piedade. Jaboatāo, PE. CEP: 54450-020. 
va a atividade anticoagulante da heparina, nesses grupos, e evidenciaram que a necessidade de heparina e sua percentagem de degradação variavam de paciente para paciente, sugerindo que um protocolo padrăo poderia incorrer no erro de sub ou superheparizinar os doentes. Estes autores estabeleceram, entāo, uma medida simples de determinar a curva de heparinização dos pacientes através do TCA (1),

O mecanismo de eliminação da heparina, segundo OLSSON et al. (4), varia de individuo para individuo e depende, também, da dose administrada. Ademais, HORKAY et al. (5), analisando a resposta da heparinização em adultos e crianças submetidos à cirurgia cardíaca com CEC observaram que a concentração de heparina nas crianças declina mais rapidamente.

Os métodos empregados na monitorizaçăo da quantidade de heparina circulante durante a CEC baseiam-se, essencialmente, na atividade anticoagulante deste composto. Esses métodos nāo identificam as heparinas de baixo peso molecular que fazem parte das preparaçōes comerciais. Ainda, a heparina injetada pode eventualmente ser degradada a fragmentos menores, que também nāo exibiriam atividade anticoagulante. A proposiçāo do presente trabalho é, através da extraçăo da heparina, por métodos físico-químicos, esclarecer como se comporta o nível sérico da heparina e sua atenuação em função do tempo, em pacientes submetidos à revascularização do miocárdio com CEC. Esses métodos desenvolvidos por DIETRICH et al. ${ }^{(6-8)}$, CASSARO \& DIETRICH ${ }^{(9)}$ e JAQUES et al. (10) independem da atividade anticoagulante da molécula e são capazes de quantificar pequenas quantidades de heparina.

\section{CASUISTICA E MÉTODOS}

Foram dosados os niveis sóricos de heparina em 27 pacientes submetidos à revascularização do miocárdio com CEC, no período de julho a novembro de 1993. Vinte eram do sexo masculino e 7 do teminino, variando a idade entre 37 e 79 anos (média de 61 anos). Dentre os critérios para fazer parte do protocolo estavam: ser portador de insuficiência coronária; ser a primeira operação cardiaca; não estar fazendo uso de heparina; apresentar coagulograma, função renal e função hepática normais. $O$ objetivo seria manter o grupo o mais homogênio possivel.

Todos os pacientes foram operados através de esternotomia mediana auxílio da circulação extracorpórea convencional. A heparinizaçăo foi feita por infusāo de $400 \mathrm{UI} / \mathrm{kg}$ de heparina (Organon) no átrio direito. Metade desta dose foi repetida a cada hora, enquanto durou a CEC.
Em todos os casos utilizou-se hipotermia sistêmica moderada a $28^{\circ} \mathrm{C}$.

O tempo de CEC variou de 60 a 145 minutos (média de 101 minutos) e o tempo de pinçamento da aorta de 40 a 120 minutos (média de 78 minutos).

A primeira coleta foi obtida de cateter venoso central, 5 minutos após a heparinizaçăo. As subseqüentes, obtidas da linha arterial do circuito de circulaçăo extracorpórea, foram feitas após 10 e 30 minutos da heparinização $e$, novamente, a cada 30 minutos, enquanto durou a perfusāo. Finalmente, a última coleta, também obtida do cateter venoso central, foi feita 10 minutos após a infusāo de protamina. A sua dose foi calculada multiplicando-se por 1,5 a dose total de heparina administrada durante a operação.

A identificação e a quantificaçăo da heparina extraída do sangue foram teitas por microeletroforese em gel de agarose, de acordo com o método de JAQUES et al. (10) e as modificaçōes de DIETRICH \& DIETRICH ${ }^{(6)}$, O erro do método é de aproximadamente $5 \%$.

\section{ANÁLISE ESTATISTICA}

Média, erro-padrão e desvio padrão das variáveis quantitativas foram calculadas. Além disso, foram também calculados o coeficiente de correlaçăo entre a concentraçāo sérica de heparina, a idade, o peso e a altura. A comparaçāo dos dados entre os dois sexos foi feita através do teste "T". A análise de regressăo foi usada para estudar o efeito do tempo sobre a concentraçăo de heparina. As comparaçōes das concentraçōes nos diversos tempos dos mesmos pacientes foram realizadas através do teste "T" pareado, por tratar-se dos mesmos individuos.

Para se eliminar a influència do peso, visto que a dosagem inicial baseia-se nesta variável, foram calculados os valores relativos ao tempo- 5 minutos - para todos os outros tempos através da fórmula: V.R. $=(100 \times T n): T 5$, onde " $n$ " varia de 5 a 150.

\section{RESULTADOS}

A Tabela 1 mostra os valores absolutos da concentração de heparina $(\mu \mathrm{g} / \mathrm{ml})$ nos respectivos tempos ( $\mathrm{min}$ ). As médias e desvios-padrão das variáveis especificas estāo apresentadas na Tabela 2 e as referentes às concetraçōes da heparina na Tabela 3. O Gráfico 1 representa as médias dos valores absolutos, com os respectivos erros-padrăo. O Gráfico 2 mostra os mesmos parâmetros, baseados nos valores relativos ao tempo 5 minutos. 
Moraes F, Nader H, Dietrich C P, Buffolo E - Dosagem da heparina em cirurgia cardiaca com circulaçāo extracorpórea. Rev Bras Cir Cardiovasc 1996; 11 (3): 201-7

TABELA 1

PACIENTES SEGUNDO NÚMERO DO CASO, INICIAIS E VALORES ABSOLUTOS DA CONCENTRAÇĀO DE HEPARINA $(\mu \mathrm{g} / \mathrm{m} /)$ NOS RESPECTIVOS TEMPOS $(\mathrm{min})$

\begin{tabular}{|c|c|c|c|c|c|c|c|c|c|}
\hline CASOS & INICIAIS & $T 5$ & $T 10$ & T30 & $T 60$ & T90 & $T 120$ & T150 & Pós prota \\
\hline 01 & AP & 16,28 & 10,10 & 10,26 & 6,70 & 10,40 & & & 0,14 \\
\hline $02^{\circ}$ & $\mathrm{FA}$ & 8,52 & 11,20 & 8,12 & 7,36 & 10,52 & & & 0,32 \\
\hline $03^{* *}$ & $\mathrm{FJ}$ & 11,4 & 8,42 & 10,86 & 9,0 & & & & 0,30 \\
\hline 04 & $\mathrm{MJ}$ & 13,7 & 12,25 & 7,20 & 6,85 & 12,40 & 8,30 & 9,70 & 0,73 \\
\hline 05 & BS & 11,92 & 10,64 & 9,30 & 9,04 & 15,0 & & & 0.28 \\
\hline 06 & ML & 11,70 & 11,66 & 9,46 & 9,40 & 12,20 & & & 0,36 \\
\hline 07 & EA & 16,80 & 14,80 & 10,14 & 8,58 & 15,84 & 12,44 & & 0,26 \\
\hline 08 & WR & 14,86 & 12,66 & 11,72 & 11,60 & 13,80 & & & 0,11 \\
\hline 09 & JB & 12,64 & 10,42 & 10,08 & 9,22 & 9,38 & & & 0,33 \\
\hline 10 & $A B$ & 12,32 & 10,62 & 8,76 & 8,80 & 10,68 & & & 0,20 \\
\hline 11 & GC & 20,0 & 13,10 & 12,70 & 9,50 & 23,90 & & & 1,28 \\
\hline 12 & $\mathrm{JB}$ & 10,64 & 10,62 & 9,34 & 7,80 & 10.70 & & & 0,42 \\
\hline 13 & WM & 20,98 & 20,64 & 18,60 & 17,32 & & & & 0,93 \\
\hline 14 & $\mathrm{JF}$ & 16,46 & 13.26 & 10,28 & 8,68 & 14,22 & 5,08 & & 0,17 \\
\hline 15 & IP & 30,0 & 25,90 & 23,28 & 14,82 & 21,36 & 22,12 & & 2,30 \\
\hline 16 & NB & 21,84 & 18,40 & 16,78 & 15,92 & 14,48 & & & 2,30 \\
\hline 17 & $\mathrm{BC}$ & 17,10 & 13,80 & 13,94 & 8,52 & 20,64 & & & 0,52 \\
\hline 18 & DB & 17,24 & 15,42 & 14,36 & 9,36 & 16,70 & & & 1,14 \\
\hline 19 & JA & 13,46 & 8,84 & 6,66 & 6,02 & 13,80 & & & 1,09 \\
\hline 20 & $\mathrm{JL}$ & 25,38 & 25.14 & 23,48 & 22,72 & 30,68 & & & 1,25 \\
\hline 21 & $C D$ & 14,24 & 13,18 & 11,50 & 10,72 & & & & 0,55 \\
\hline 22 & $\mathrm{HC}$ & 13,16 & 12,96 & 11,16 & 10,38 & 11,66 & & & 0,37 \\
\hline 23 & $\mathrm{AL}$ & 16,28 & 15,46 & 12,12 & 11,26 & 15,06 & & & 1,10 \\
\hline 24 & RA & 11,30 & 10,0 & 10,5 & 9,30 & 12,70 & 12,10 & 6,60 & 0,96 \\
\hline 25 & JO & 15,62 & 14,54 & 9,02 & 8,12 & 13,80 & & & 0,97 \\
\hline 26 & MA & 14,08 & 13,44 & 12,92 & 12,92 & 14,56 & & & 0,10 \\
\hline 27 & ME & 12,56 & 12,10 & 9,20 & 9,40 & 9,86 & & & 0,32 \\
\hline
\end{tabular}

- Acrescentado sangue mais heparina ao priming do oxigenador.

* Acrescentado sangue mais heparina ao priming do oxigenador, além de ter sido iniciada a CEC após 10 minutos da heparinização, por problemas técnicos.

TABELA 2

MÉDIASE DESVIOS.PADRÄO DAS VARIÁVEIS ESPECIFICAS RELATIVAS A CIRURGIA DOS PACIENTES INCLUIDOS NA AMOSTRA $(n=27)$

\begin{tabular}{lrrc}
\hline VARIÁVEL & MÉDIA & $\begin{array}{r}\text { DESVIO } \\
\text { PADRĀO }\end{array}$ & INTERVALO \\
\hline Idade (anos) & 60,74 & 8,97 & $(37-79)$ \\
Peso (kg) & 71,40 & 14,05 & $(44-115)$ \\
Altura (cm) & 165,26 & 7,37 & $(153-178)$ \\
T. CEC (min) & 100,59 & 21,60 & $(60-145)$ \\
T. Anóxia (min) & 78,04 & 19,69 & $(40-120)$ \\
\hline
\end{tabular}

\section{TABELA 3}

MÉDIAS E DESVIOS-PADRĀO DAS CONCENTRACOOEES DE HEPARINA NOS DIFERENTES TEMPOS CONSECUTI VOS DE COLETA

\begin{tabular}{lrrrr}
\hline VARIAVEL & $\boldsymbol{n}$ & MÉDIA & DESVIO & $\begin{array}{c}\text { INTERVALO } \\
\text { PADRĂOO }\end{array}$ \\
\hline T5 & 27 & 15,57 & 4,74 & $(8,52-30,00)$ \\
T10 & 27 & 13,67 & 4,35 & $(8,42-25,90)$ \\
T30 & 27 & 11,92 & 4,24 & $(6,66-23,48)$ \\
T60 & 27 & 10,34 & 3,65 & $(6,02-22,72)$ \\
T90 & 24 & 14,77 & 4,99 & $(9,38-30,68)$ \\
T120 & 5 & 12,01 & 6,41 & $(5,08-22,12)$ \\
T150 & 2 & 8,15 & 2,19 & $(6,60-9,70)$ \\
Pósprotamina & 27 & 0,70 & 0,60 & $(0,10-2,30)$ \\
\hline \multicolumn{5}{r}{}
\end{tabular}

GRÁFICO 1

MEDIAS E ERROS.PADRÄO DAS CONCENTRACYÓES $(\mu \mathrm{G} / M L)$ SANGÜINEAS DE HEPARINA NOS TEMPOS (MIN) PRÉ-ESTABELECIDOS.

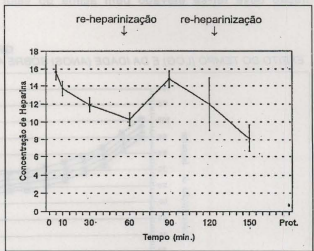

A comparaçāo entre os valores da concentração de heparina no tempo 5 e no tempo 60, através do teste " $T$ " pareado, revela que a concentraçăo de heparina 1 hora após a aplicaçăo é de aproximada- 
GRÁFICO 2

MÉDIAS E ERROS-PADRAOO DOS VALORES PERCENTUAIS DA CONCENTRACÃO SANGUINNEA DE HEPARINA NOS TEMPOS (MIN) PRÉ-ESTABELECIDOS.

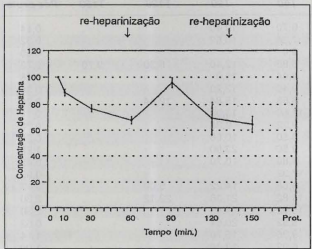

mente $68 \%$ da encontrada aos 5 minutos $(T=22,82$; $p=0$ ). Após a administraçāo da protamina, a concentraçāo de heparina se reduz a $4 \%$ do valor inicial $(\mathrm{t}=8,15 ; \mathrm{p}=0)$.

Observando-se os gráficos 1 e 2, pode-se ver que, aos 90 minutos, ou seja, 30 minutos após a administração de metade da dose de heparina, o valor médio está $30 \%$ acima do valor encontrado para 30 minutos, sendo significante a média das diferenças, calculada através do teste " $\mathrm{T}$ " pareado: $3,06(T=4,72: p<0,001)$. Isso mostra que, logo após a administraçăo da segunda dose, a concentração deve ter-se elevado bem acima do valor encontrado aos 5 minutos, o que também é comprovado pela média do tempo 90 , a qual năo difere significantemente da média do tempo $5: 0,81(\mathrm{t}=$ $1,19 ; p=0,25)$. Portanto, meia hora após a administração da segunda dose encontra-se praticamente a mesma concentração que 5 minutos após a primeira aplicaçāo $(96 \%)$.

A análise de regressăo dos valores da concentraçăo de heparina nos tempos $5,10,30$ e 60 minutos sobre o tempo (em logarítmo, desde que sua influência segue essa distribuição) mostrou um resultado significante $(F=22,78 ; p<0,001)$, originando a seguinte equação de regressão: Conc. Heparina = $18,65-2,023 \times$ minutos $(\mathrm{In})$.

Realizou-se a análise de regressão múltipla com os valores relativos das concentraçōes de heparina sobre o tempo (In) e a idade (as outras variáveis especificas não tiveram influência significante). A Tabela 4 mostra os resultados dessa regressão.

O resultado dessa regressāo gerou a seguinte equação:

Conc. Heparina $=104,7+(-12,85 \times$ minutos $($ In) $)+0,25 \times$ idade

Através desta equaçăo foram estimados os valores esperados para as idades representadas no Gráfico 3 , onde se observa que quanto maior a idade maior a concentração de heparina no sangue.

As correlações entre as concentraçōes de heparina $e$ as variáveis específicas não foram estatisticamente significantes, com exceção do peso, que mostrou uma correlaçăo significante no tempo 5 ( $\mathrm{r}$ $=0,42 ; p=0,02$ ), o que era de se esperar, desde que a dose administrada é calculada em funçăo do peso.

GRÁFICO 3

EFEITO DO TEMPO (LOG) E DA IDADE (ANOS) SOBRE A CONCENTRAÇĀO RELATIVA (\%) DA HEPARINA NO SANGUE.

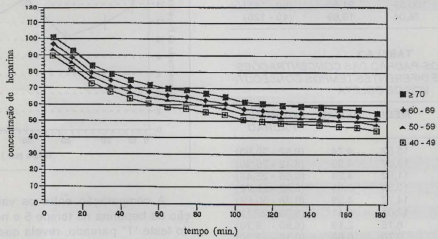


TABELA 4

VALORES OBTIDOS ATRAVESS DA ANÁLISE DE REGRESSĀO MÚLTIPLA

\begin{tabular}{lrrrr}
\hline $\begin{array}{l}\text { VARIÁVEL } \\
\text { INDEPENDENTE }\end{array}$ & COEFICIENTE & ERRO-PADRÄO & TESTE & PROBABILIDADE \\
\hline Constante & 104.755013 & 7.438931 & 14.0820 & 0.00 \\
Minutos (In) & -12.855361 & 1.153207 & -11.1475 & 0.00 \\
Idade & 0.251593 & 0.110123 & 2.2847 & 0.03 \\
\hline
\end{tabular}

Não houve influència do sexo nos niveis de heparina no sangue.

\section{COMENTÁRIOS}

O processo de heparinizaçăo e de sua neutralizaçăo, estabelecido para este trabalho, difere do preconizado por BULL et al. (1). Estabeleceu-se que a heparinização seria feita com $400 \mathrm{Ul} / \mathrm{kg}$ e, a cada hora, metade da dose seria administrada, independente do TCA, ou de outro método de avaliação do estado de coagulabilidade. No método defendido por BULL et al. (1), a heparinização baseia-se no TCA, ou seja, não se mantém uma heparinização padronizada. Ademais, os pacientes apresentam tolerâncias individuais (11) $\mathrm{e}, \mathrm{com}$ isso, as doses variam de doente para doente, o que difilcutaria a aplicaçåo do protocolo utilizado neste estudo. O critério para a neutralizaçāo com sulfato de protamina foi também fixo, ou seja, 1,5 miligramas de protamina foram administrados para cada 100 UI de heparina injetada durante a operaçăo.

Os métodos de heparinizaçāo e neutralizaçāo da heparina dos pacientes submetidos à cirurgia cardiovascular com CEC são muito variáveis (1, 12-14). Ressalta-se, contudo, que não se encontrou na literatura nenhum trabalho que tenha analisado o comportamento da concentraçăo sérica de heparina em funçāo do tempo e da dose administrada, independente do estado de coagulabilidade dos pacientes.

Uma das questōes ainda não respondidas seria: qual o tempo necessário, após a administração da heparina, para que o paciente esteja efetivamente anticoagulado, sem o risco de formaçāo de coágulos nos circuitos da CEC. BOUNAMEAUX et al. (11) defenderam a existência de uma tolerância individual e afirmaram que o nivel de anticoagulaçăo năo está apenas relacionado com a concentração plasmática de heparina. EFFENEY et al. (15) utilizaram o TCA, mas somente 20 minutos após a infusão de heparina. Observou-se no presente estudo que, à exceção dos casos 2 e 3 , todos os pacientes apresentaram, aos 5 minutos após a administraçăo de heparina, concentraçāo sangüínea máxima. No caso de número 2 isto năo ocorreu porque adicionou-se, ao priming do oxigenador, sangue com heparina. No caso de núme- ro 3 repetiu-se o fato, mas, por problemas técnicos, atrasou-se o início da circulação extracopórea.

Ao verificar que ao tempo de 5 minutos encontrou-se uma concentração máxima de heparina, foram estabelecidos valores porcentuais para as concentraçōes subseqüentes em relação ao tempo 5 . Assim, os valores referentes ao tempo de 5 minutos foram admitidos como sendo $100 \%$. Pode-se observar no Gráfico 2 como se comportaram a curva das médias das concentrações de heparina (em valores percentuais) e os erros-padrāo nos tempos de 5 , $10,30,60,90,120$ e 150 minutos e após a administraçăo de protamina.

Quando foram comparadas as concentraçōes de heparina nos diversos tempos, verificou-se que - seu nivel sérico (em média), 1 hora após sua administraçăo, foi em torno de $68 \%$ do encontrado no tempo de 5 minutos. ESTES \& POULIN (16) mostraram que $50 \%$ da concentraçāo sangüinea de heparina, quando administrados $100 \mathrm{UI} / \mathrm{kg}$, podem ser encontrados 90 minutos após sua administraçăo, assim como a atividade anticoagulante da heparina, que foi de 1 hora em meia. Segundo OLSSON et al. (4), quando administrada essa mesma concentração de heparina, a meia-vida da atividade anticoagulante foi de 56 minutos.

Através da análise de regressão nos tempos 5 , 10,30 e 60 minutos, evidenciou-se, como mostra o Gráfico 3, que, para se obter $50 \%$ da concentração de heparina encontrada aos 5 minutos, seriam necessários 215 minutos, aproximadamente.

Após 1 hora da primeira injeção de heparina, repetiu-se o procedimento, infundindo metade da dose. Ao observar os Gráficos 1 e 2, verifica-se que, 30 minutos após a segunda dose, a concentraçăo de heparina está praticamente igual à do tempo de 5 minutos (96\%). Através desse achado, pode-se sugerir que houve uma super-heparinização, quer dizer, a concentração de heparina, logo após a segunda dose, ultrapassou a concentração obtida no tempo 5. Julga-se, portanto, que a concentraçāo de heparina no tempo 90 deveria ser próxima da encontrada no tempo 30 , para que mantivesse uma concentração homogênea, porém o valor achado é $30 \%$ superior ao estimado, tendo sido significante a média das diferenças. 
Analisando a resposta da hiperinizaçāo em adultos e crianças submetidos à cirurgia cardiaca com CEC, HORKAY et al. (5) observaram que a concentração de heparina nas crianças declina mais rapidamente que nos aduitos.

Neste estudo, apesar dos pacientes serem todos adultos, mas com idades variadas, observou-se uma correlaçăo entre a idade $e$ a concentração de heparina nos respectivos tempos. Observando o Gráfico 4 percebe-se que quanto maior a idade maior a concentração da heparina no sangue, ou seja, os pacientes mais idosos parecem eliminar ou metabolizar a heparina mais lentamente. Apesar desse achado já ter sido relatado na literatura, pesquisas mais aprofundadas nesses pacientes poderiam trazer resultados mais seguros, visto que no presente estudo o número de casos năo foi tăo significativo.

Ao correlacionar as variáveis especificas com a concentração de heparina, observou-se que o peso mostrou uma correlação significante com o tempo de 5 minutos. Isso, possivelmente, deveu-se ao fato das doses administradas serem calculadas de acordo com o peso do paciente. O fato dos pacientes mais pesados apresentarem correlaçăo nos tempos subsequjentes sugere que 0 "excesso" de heparina poderia estar sendo armanezado no compartimento extravascular (16-18), ou estar sendo eliminado pela urina $(16,19)$.

Ao analisar a média das concentrações de heparina, colhida 10 minutos após o término da administraçăo de protamina, observou-se um valor médio de $4 \%$ da concentração de heparina encontrada no tempo 5. Segundo o trabalho de GARCIA et al (12) foi evidenciado, nesse mesmo tempo, $10 \%$ da con- centração de heparina encontrada aos 10 minutos após a heparinização. Nesse trabalho os autores utilizaram o método de heparinizaçāo e neutralizaçāo descrito por BULL et al (1). Estes resultados sugerem que o método de neutralização de heparina (1.5:1) empregado no presente trabalho promove neutralização mais eficiente.

A complexidade estrutural da molécula da heperina, associada à sua complicada ação farmacodinâmica, a torna uma substância com características peculiares e de difícil compreensão, no que diz respeito à sua atuaçăo no organismo humano.

Apesar de descoberta há muitos anos, ainda persiste uma série de questionamentos, no que diz respeito à açāo desta droga sobre o sistema de coagulação. Acredita-se que a complexidade de seus efeitos, a diversidade das preparações, com as concentrações as mais variadas possíveis (heparinas de alto e baixo peso molecular) (20), associadas a protocolos diferentes $\theta$ a tolerâncias individuais diversas (11-16) têm contribuído para retardar 0 estabelecimento de um consenso sobre a farmacodinåmica da heparina. Principalmente no que diz respeito ao seu uso em cirurgia cardiovascular, quando, devido aos circuitos de circulaçāo extracorpórea, necessitou-se doses elevadas.

0 presente trabalho, utilizando originalmente o método de dosagem do nível sérico da heparina durante operaçōes de revascularização do miocárdio, possibilitou estabelecer o comportamento da curva de heparinização durante a circulação extracorpórea e sua atenuação no tempo, além de verificar a concentraçăo residual de heparina após a neutralizaçăo com sulfato de protamina.

\section{RBCCV $44205-310$}

Moraes F, Nader H, Dietrich C P. Buffolo E-Measurement of heparin in cardiac surgery with extracorporeal circulation. Rev Bras Cir Cardiovasc 1996; 11 (3): 201-7.

ABSTRACT: In order to quantify seric levels of heparin, its attenuation as a time function and its residual value after neutralization with protamine sultate, blood samples were collected, at pre-set intervals, from 27 patients undergoing myocardial revascularization surgery under extracorporeal circulation. After heparinization (400 Ul/Kg), blood samples were collected at 5, 10,30 and 60 minutes and subsequently every 30 minutes depending upon the extracorporeal circulation lenght. At each hour after heparinization, a new heparin dose $\left(200 \mathrm{UI} / \mathrm{Kg}\right.$ ) was administered. The samples were kept at $4^{\circ} \mathrm{C}$ prior to the heparin extract process, which was performed by physical/chemical method. The dosages showed that 5 minutes after heparinization the patients show maximal blood concentration of heparin and after 60 minutes it is aproximately $68 \%$ of the concentration at 5 minutes. At 90 minutes time, that is, after re-heparinization, the concentration of heparin is $96 \%$ of the one showed on the fifth minute and after the protamine sulfate neutralization (1.5:1), a residual value corresponding to $4 \%$ of the one initially observed is still found. It was observed that older patients have a tendency to keep longer seric heparin level, and heparin concentration at a given time could be estimated by the Equation Heparin - Concentration $=104.7+(-12.85 \times$ minutes $(\ln )+0.25 \times$ age $)$.

DESCRIPTORS: Heparin, blood. Myocardial revascularization. Extracorporeal circulation. 


\section{REFERÊNCIAS BIBLIOGRÁFICAS}

1 Bull B S, Korpan R A, Huse W M, Briggs B D - Heparin therapy during extracorporeal circulation: I. Problems inherent in existing heparin protocois. J Thorac Cardiovasc Surg 1975; 69: 674-84.

2 Abildgaard U - Highly purified antithrombin III with heparin cofator activity prepared by disc electrophoresis. Scand J Clin Lab 1968; 21: 89-91.

3 Hattersley P G - Activated coagulation time of whole blood. JAMA 1966; 196: 436-40.

4 Olsson P. Lagergren $\mathrm{H}$, Stig E K - The elimination from plasma of intravenous heparin: an experimental study on dogs and humans. Acta Med Scand 1963; 173: 619-30.

5 Horkay F, Martin P. Rajah M, Walker D - Response to heparinization in adults and childern undergoing cardiac operations. Ann Thorac Surg 1922; 53: 822-6.

6 Dietrich C P \& Dietrich S M C - Electrophoretic behavior of acidic mucopolysaccharides in diamine buffers. An Biochem 1976; 70: 645-7.

7 Dietrich C P, McDutfie N M, Sampaio L O - Identification of acidic mucopolysaccharides by agarose gel electrophoresis. J Chromatogr 1977; 130: 299-304.

8 Dietrich C P. Tersariol I L S, Silva R G, Bianchini P, Nader H B - Dependence of the C-6 sulfate of the glucosamine moiety and 1-4 glycosydic linkage of heparin disaccharides for production of hemorrhage: reversal of the antihemostatic activity of heparin and their fragments by adenosin triphosphate and myosin. Semin Thromb Hemost 1991; 17: 65-73.

9 Cassaro C M F \& Dietrich C P - The distribution of sulfated mucopolysaccharides in invertebrates. I Biol Chem 1977; 252: 2254-61.

10 Jaques $L B$, Ballieux $R E$, Dietrich $C P$, Kavanagh $L W$ - A microeletrophoresis method for heparin. Can J Physiol Pharmacol 1968; 46: 351-60.
11 Bounameaux $\mathrm{H}$, Marbet G A, Lammle B, Eichlisberger $R$, Duckert F - Monitoring of heparin treatment: comparison of thrombin time, activated partial thromboplastin time, and plasma heparin concentration, and analysis of the behaviour of antithrombin III. Am J Clin Pathol 1980; 74: 68-73.

12 Garcia H V, Buffolo E, Nader H B, Dietrich C P. ATP reduces blood loss produced by heparin in cardiopulmonary bypass operations. Ann Thorac Surg 1994; 57: $956-9$.

13 Jaberi M, Bel W R, Benson D W - Control of heparin therapy in open-heart surgery. J Cardiovasc Surg $1974 ; 67: 133-41$.

14 Kaul T K, Crow M J, Rajah S M, Deverall P B, Watson D A - Heparin administration during extracorporeal circulation: heparin rebound and postoperative bleeding. J Thorac Cardiovasc Surg 1979; 78: 95-102.

15 Effeney D J, Goldstone J, Chin U, Krupski W C, Ellis $\mathrm{R} \mathrm{J}$-Intraoperative anticoagulation in cardiovascular surgery. Surgery 1981; 90: 1068-74.

16 Estes J W \& Poulin P F - Pharmacokinetics of heparin. Thrombos Diathes Haemorrh (Stuttgard) 1974;33: 26-37.

17 Bârzu T, Molho $P$, Tobelem G, Petitou M, Caen J P Binding of heparin and low molecular weight heparin fragments to human vascular endothelial cells in culture. Nouv Rov Fr Hematol 1984; 26: 243-7.

18 Swart C A M, Nijmeyer B, Roelofs J M M, Sixma $\mathrm{J}$ J Kinetics of intravenously administered heparin in normal humans. Blood 1962; 60: 1251-8.

19 Boldt J, Zickmann B, Herold C, Scholz S, Dapper F, Hempelmann G - Heparin management during cardiac surgery with respect to various blood-conservation techniques. Surgery 1992; 111: 260-5.

20 Nader H B, Dietrich C P, Strauss A H, Takahashi H K - Características físico-químicas da heparina relacionadas com a açāo anticoagulante e anti-hemostática. Rev Bras Biol 1979; 39: 793-816. 